\title{
Stratification and prediction of remission in first-episode psychosis patients: the OPTiMiSE cohort study
}

Emanuela Martinuzzi', Susana Barbosa', Douglas Daoudlarian (1)', Wafa Bel Haj Ali², Cyprien Gilet', Lionel Fillatre², Olfa Khalfallah", Réjane Troudet ${ }^{2}$, Stéphane Jamain $\mathbb{1}^{2}$, Guillaume Fond ${ }^{4}$, Iris Sommer, ${ }^{5,6}$, Stefan Leucht7,8, Paola Dazzan ${ }^{8}$, Philip McGuire ${ }^{8}$, Celso Arango ${ }^{9}$, Covadonga M. Diaz-Caneja ${ }^{9}$, Wolfgang Fleischhacker ${ }^{10}$, Dan Rujescu ${ }^{11}$, Birte Glenthøj ${ }^{12}$, Inge Winter ${ }^{13}$, René Sylvain Kahn ${ }^{13}$, Robert Yolken ${ }^{14}$, Shon Lewis ${ }^{15}$, Richard Drake $\mathbb{B}^{15}$, Laetitia Davidovic', Marion Leboyer ${ }^{2,16,17}$ and Nicolas Glaichenhaus ${ }^{1,17}$, the OPTiMiSE Study Group

\begin{abstract}
Early response to first-line antipsychotic treatments is strongly associated with positive long-term symptomatic and functional outcome in psychosis. Unfortunately, attempts to identify reliable predictors of treatment response in firstepisode psychosis (FEP) patients have not yet been successful. One reason for this could be that FEP patients are highly heterogeneous in terms of symptom expression and underlying disease biological mechanisms, thereby impeding the identification of one-size-fits-all predictors of treatment response. We have used a clustering approach to stratify 325 FEP patients into four clinical subtypes, termed C1A, C1B, C2A and C2B, based on their symptoms assessed using the Positive and Negative Syndrome Scale (PANSS) scale. Compared to C1B, C2A and C2B patients, those from the C1A subtype exhibited the most severe symptoms and were the most at risk of being non-remitters when treated with the second-generation antipsychotic drug amisulpride. Before treatment, C1A patients exhibited higher serum levels of several pro-inflammatory cytokines and inflammation-associated biomarkers therefore validating our stratification approach on external biological measures. Most importantly, in the C1A subtype, but not others, lower serum levels of interleukin (IL)-15, higher serum levels of C-X-C motif chemokine 12 (CXCL12), previous exposure to cytomegalovirus (CMV), use of recreational drugs and being younger were all associated with higher odds of being non-remitters 4 weeks after treatment. The predictive value of this model was good (mean area under the curve $(A \cup C)=0.73 \pm 0.10$ ), and its specificity and sensitivity were $45 \pm 0.09 \%$ and $83 \pm 0.03 \%$, respectively. Further validation and replication of these results in clinical trials would pave the way for the development of a blood-based assisted clinical decision support system in psychosis.
\end{abstract}

Correspondence: Marion Leboyer (marion.leboyer@inserm.fr) or Nicolas Glaichenhaus (nicolas.glaichenhaus@unice.fr)

'Université Côte d'Azur, Centre National de la Recherche Scientifique, Institut de Pharmacologie Moléculaire et Cellulaire, Valbonne, France

${ }^{2}$ Université Paris Est Créteil, Faculté de Medicine Institut, National de la Santé et de la Recherche Médicale, Créteil, France

Full list of author information is available at the end of the article.

These authors contributed equally: Emanuela Martinuzzi, Susana Barbosa, Douglas Daoudlarian, Wafa Bel Haj Ali

Co-senior authors: Marion Leboyer, Nicolas Glaichenhaus.

\section{Introduction}

Psychotic symptomatology includes loss of contact with reality, thought disorder, delusions and hallucinations, unusual or bizarre behavior, impaired social interactions and difficulties to carry out daily activities ${ }^{1}$. While psychosis could be caused by recreational drug use, physical illness or brain trauma, it is often symptomatic of the onset of severe psychiatric disorders such as schizophrenia, schizoaffective disorder or bipolar disorder.

\section{(c) The Author(s) 2019}

(c) Open Access This article is licensed under a Creative Commons Attribution 4.0 International License, which permits use, sharing, adaptation, distribution and reproduction cc) in any medium or format, as long as you give appropriate credit to the original author(s) and the source, provide a link to the Creative Commons license, and indicate if changes were made. The images or other third party material in this article are included in the article's Creative Commons license, unless indicated otherwise in a credit line to the material. If material is not included in the article's Creative Commons license and your intended use is not permitted by statutory regulation or exceeds the permitted use, you will need to obtain permission directly from the copyright holder. To view a copy of this license, visit http://creativecommons.org/licenses/by/4.0/. 
According to current guidelines, first-line treatments of psychosis involve the use of the minimum effective dose of second-generation antipsychotics whenever possible. Whatever criteria are used to assess response to treatment, responses are highly heterogeneous. While 25-30\% of first-episode psychosis (FEP) patients fully respond, a majority respond partially or not at all, and are therefore switched to second-line treatments ${ }^{2}$. As early response to treatment is one of the main factors associated with improved long-term prognosis ${ }^{3-5}$, identifying predictors of treatment response in FEP patients is an important issue in the field ${ }^{6}$. Response to treatment could be assessed either using predefined cutoffs in the percentage of reduction of baseline scores on a psychopathology rating $\mathrm{scale}^{7}$, or by measuring the proportion of patients meeting remission criteria. According to the definition proposed by the Remission in Schizophrenia Working Group (RSWG), remission can be defined by an absolute threshold of severity of symptoms in three dimensions: reality distortion, disorganization and negative symptoms ${ }^{8}$. Using this consensus definition, it was found that global functioning in the year before admission, the total score of the Strauss Carpenter Prognostic Scale and the Positive and Negative Syndrome Scale (PANSS) negative sub-score at admission were all predictive of symptom remission in cohorts of schizophrenia inpatients ${ }^{9,10}$. Despite these latter studies, clinicians still lack reliable predictors of remission in FEP patients.

Several environmental risk factors for psychosis have been identified $^{11}$ including autoimmune disorders ${ }^{12}$ and infection with Toxoplasma gondii ${ }^{13}$, cytomegalovirus $(\mathrm{CMV})^{14}$ and herpes simplex virus (HSV) type $1^{15}$. Meta-analyses have shown that drug-naive FEP patients exhibit altered serum levels of various cytokines compared to healthy individuals ${ }^{16-18}$. Since these data suggested a possible link between immune dysregulation and psychosis, it was proposed that serum levels of cytokines, chemokines and biomarkers of inflammation could predict early response to treatment ${ }^{6}$. However, antipsychotic treatments could impact cytokine levels including those of interleukin (IL)$1 \beta$, interferon (IFN)- $\gamma$, IL-12 and tumor necrosis factor (TNF)- $\alpha^{19-23}$. Therefore, the predictive value of serum biomarkers should be ideally assessed in minimally treated or untreated FEP patients, which is a challenge because of the difficulty to enroll these patients in clinical trials. This may be the reason for which only a few studies have investigated the association between baseline levels of peripheral biomarkers and remission in FEP patients. To identify biological predictors of remission in FEP patients, we have analyzed clinical data and biological samples from the multinational, multi-centered, randomized, doubleblind "Optimization of Treatment and Management of Schizophrenia in Europe (OPTiMiSE)" study in which FEP patients were clinically assessed before and after 4 weeks of treatment with the second-generation antipsychotic amisulpride $^{24}$. Our results demonstrate that serum levels of immune-related proteins before treatment combined with a few clinical variables could predict remission in at least a subtype of FEP patients.

\section{Materials and methods \\ Patients}

The OPTiMiSE study was conducted in 27 general hospitals and clinics in 14 European countries, Israel and Australia (Clinicaltrials.gov identifier is NCT01248195). FEP patients based on the EUFEST (European First Episode Schizophrenia Trial) study definition ${ }^{25}$ were recruited between May 2011 and April 2016 at the participating centers from nearby healthcare facilities. Eligible patients were aged 18-40 years and met criteria of the Diagnostic and Statistical Manual of Mental Disorders (4th edition) for schizophrenia, schizophreniform disorder or schizoaffective disorder. A total of 479 patients signed informed consent. Diagnoses were confirmed by the Mini International Neuropsychiatric Interview plus. Patients were excluded if more than 2 years had passed since the start of the FEP; if any antipsychotic drug had been used for more than 2 weeks in the previous year and/or for a total of 6-week lifetime; if patients had a known intolerance to one of the study drugs; if patients met any of the contraindications for any of the study drugs; if patients were coercively treated and/or represented by a legal guardian or under legal custody; or if patients were pregnant or breast feeding. Patients were required to provide written informed consent.

\section{Patient clinical assessment and primary outcome}

A screening visit was conducted during which eligibility was assessed. Baseline data were obtained regarding demographics, diagnoses, current treatments and psychopathology: PANSS total score and sub-scores, overall severity of symptoms assessed using the Clinical Global Impression (CGI) scale ${ }^{26}$, depression assessed using the Calgary Depression Scale for Schizophrenia (CDSS $)^{27}$ and social functioning assessed using the Personal and Social Performance Scale (PSP) ${ }^{28}$. Recreational drug use was also assessed. Data were collected at baseline and 4-5 weeks later.

All patients were treated for 4 weeks with up to $800 \mathrm{mg} /$ day amisulpride in an open design. The primary outcome was symptomatic remission according to the criteria of Andreasen et al. ${ }^{8}$ : a score of $\leq 3$ (on a scale ranging from 1 to 7) simultaneously on 8 PANSS items: P1, P2, P3, N1, N4, N6, G5 and G9.

\section{Blood samples}

Peripheral blood samples were obtained from fasting subjects between 7:00 am and 9:00 am. Five milliliters of 
peripheral blood were drawn by venipuncture into serum Vacutainer tubes. For the serum collection, the blood was allowed to clot for $1 \mathrm{~h}$ before centrifugation $(1500 \times g$, $10 \mathrm{~min})$. The serum and plasma samples were stored in $0.5 \mathrm{ml}$ aliquots at $-80^{\circ} \mathrm{C}$. For measuring protein and antibody levels, serum samples were thawed on ice, and $50 \mu \mathrm{l}$ aliquots were prepared and stored at $-80^{\circ} \mathrm{C}$.

\section{Immunoassay}

Serum levels of IL- $1 \alpha$, IL-1 $\beta$, IL-2, IL-4, IL-5, IL-6, IL-7, IL-8, IL-10, IL-12p40, IL- 12p70, IL-13, IL-15, IL-16 IL17, IL-18, IL-21, IL-23, IL-27, IFN- $\gamma$, chemokines (C-C motif chemokine ligand (CCL)-2, CCL3, CCL4, CCL11, CCL13, CCL17, CCL19, CCL20, CCL22, CCL26, CCL27, and $\mathrm{C}-\mathrm{X} 3-\mathrm{C}$ motif chemokine ligand (CX3CL)-1, CXCL10, CXCL11, CXCL12), TNF- $\alpha$, TNF- $\beta$, granulocyte macrophage-colony stimulating factor, vascular endothelial growth factor (VEGF), C reactive protein (CRP), serum amyloid A protein (SAA), soluble intercellular adhesion molecule 1 (sICAM-1) and soluble vascular adhesion molecule 1 (sVCAM-1) were measured using the Pro-inflammatory Panel 1, Cytokine Panel 1, Chemokine Panel 1, Th17 Panel 1 and Vascular Injury Panel 2 v-PLEX ${ }^{\circledR}$ kits (MSD). All assays were performed according to the manufacturer's instructions. The data were acquired on the V-PLEX ${ }^{\circledast}$ Sector Imager 2400 plate reader and analyzed using the Discovery Workbench 3.0 software (MSD). The standard curves for each cytokine were generated using the premixed lyophilized standards provided in the kits. Serial twofold dilutions of the standards were run to generate a 13-standard concentration set, and the diluent alone was used as a blank. The cytokine concentrations were determined from the standard curve using a 4-parameter logistic curve fit to transform the mean light intensities into concentrations. The lower limit of detection (LLOD) was determined for each cytokine and for each plate as the signal recorded for the blank plus 2 standard deviations (SDs).

\section{Serology}

We measured plasma immunoglobulin G (IgG) antibodies reacting to HSV type $1, \mathrm{CMV}$ and T. gondii using previously described immunoassay methods ${ }^{29}$. Diluted plasma was applied to antigens immobilized on the wells of microtiter plates and bound antibodies were quantified by means of reaction with enzyme-labeled anti-human IgG and the corresponding substrate. Reagents and assay kits for anti-HSV-1 were obtained from Focus Laboratories (USA). Anti-CMV and anti-Toxoplasma antibodies were obtained from IBL Laboratories (Germany). Results were obtained as quantitative values determined by comparison of the level of reactivity to standards run with each assay, as well as qualitative results listed as "positive" or "negative".

\section{Statistical analysis}

In univariate analysis, Mann-Whitney-Wilcoxon tests were performed to assess statistical significance of nonGaussian distributed data. To develop a predictive model for remission we used the elastic net, which is a regularized regression model, i.e., general linear model with penalties to avoid extreme parameters that could cause overfitting $^{30}$. Elastic net is also a method of selection of variables that addresses the issue of multicollinearity that arises in our dataset because cytokines and chemokines are not independent of each other. To minimize variation across testing datasets, we repeated fivefold crossvalidation 100 times with independent random dataset partitions to optimize stability ${ }^{31}$. We tuned the hyperparameters $\alpha$ and $\lambda 10$ times for each partition via fivefold cross-validation with the optimal tuning parameter values chosen to maximize the area under the receiver operating characteristics (ROC) curve (AUC) ${ }^{32}$. Weighted odds ratios (ORs) were calculated using the proportion of drawings in which the variable was selected as a weight. All statistical analyses were performed using the $\mathrm{R}$ software packages Stats $^{33}$, Caret $^{34}$, Glmnet $^{35}$, pROC $^{36}$ and eNetXplorer ${ }^{37}$.

\section{Unsupervised statistical classification}

To stratify $m$ patients into $k$ clusters based on their PANSS scores ( $d$ items per patient), we prepared a matrix $\mathrm{X}$ with one patient per line and one PANSS item per column (Supplementary Figure 1). Our objective was to find a matrix $\mathrm{Y}$ of labels. We thus tried to solve an optimization problem for finding a space which discriminated clusters based on a limited number of weighted PANSS items. The output was a W (weight) matrix with $k$ columns and $d$ lines computing the weight of each PANSS item. We achieved this goal using an alternating minimization procedure on $\mathrm{Y}$ and $\mathrm{W}$ in which we tried to minimize the Frobenius norm ${ }^{38}$.

\section{Results}

Soluble serum biomarkers did not predict remission in non-stratified FEP patients

A total of 479 patients were included in the OPTiMiSE clinical trial. Out of the 446 patients in the intention-totreat sample, 371 completed amisulpride treatment. Among those, 325 had serum samples collected before the study treatment was initiated and were included in the present study (Table 1). Clinical assessment 3-4 weeks after treatment initiation revealed that $68.6 \%$ of the patients were in symptomatic remission ${ }^{39}$ according to the consensus definition ${ }^{8}$. As a first attempt to identify biomarkers that could predict remission, we analyzed serum samples for 43 interleukins, chemokines and biomarkers of inflammation. Among these proteins, 8 were below the LLOD in more than $10 \%$ of the samples and 
Table 1 Patient clinical characteristics

\begin{tabular}{|c|c|c|c|c|c|c|c|}
\hline & \multicolumn{3}{|c|}{ Study sample as a whole } & \multicolumn{4}{|c|}{ Patient subsets } \\
\hline & All & Non-remitters & Remitters & $\mathrm{C} 1 \mathrm{~A}$ & $\mathrm{C} 1 \mathrm{~B}$ & $\mathrm{C} 2 \mathrm{~A}$ & $\mathrm{C} 2 \mathrm{~B}$ \\
\hline Number of patients & 325 & 102 & 223 & 97 & 62 & 95 & 71 \\
\hline Male (\%) & $70.2 \%$ & $73.5 \%$ & $68.6 \%$ & $63.9 \%$ & $83.9 \%$ & $63.4 \%$ & $75.3 \%$ \\
\hline Age (years) & $26.2 \pm 6.2$ & $25.0 \pm 5.6$ & $28.8 \pm 6.4$ & $25.9 \pm 5.9$ & $25.6 \pm 5.6$ & $27.0 \pm 7.0$ & $26.2 \pm 6.0$ \\
\hline BMI $\left(\mathrm{kg} / \mathrm{m}^{2}\right)$ & $23.3 \pm 4.2$ & $23.5 \pm 4.5$ & $23.2 \pm 4.1$ & $23.3 \pm 4.0$ & $23.8 \pm 3.8$ & $23.2 \pm 4.5$ & $23.0 \pm 4.5$ \\
\hline Waist circumference (cm) & $83.2 \pm 11.8$ & $84.6 \pm 11.6$ & $82.6 \pm 11.9$ & $83.2 \pm 11.8$ & $85.2 \pm 11.6$ & $82.3 \pm 12.2$ & $82.8 \pm 10.3$ \\
\hline PPANSS & $19.8 \pm 5.7$ & $21.4 \pm 5.4$ & $19.1 \pm 7.7$ & $24.6 \pm 4.1$ & $17.2 \pm 4.3$ & $21.3 \pm 4.1$ & $14.0 \pm 3.3$ \\
\hline NPANSS & $19.6 \pm 7.1$ & $22.5 \pm 7.2$ & $18.3 \pm 6.7$ & $25.7 \pm 5.0$ & $24.9 \pm 3.9$ & $14.5 \pm 4.0$ & $13.5 \pm 4.0$ \\
\hline GPANSS & $38.2 \pm 9.8$ & $40.6 \pm 8.8$ & $37.0 \pm 10.0$ & $47.8 \pm 6.8$ & $37.5 \pm 7.0$ & $36.9 \pm 6.3$ & $27.6 \pm 5.9$ \\
\hline PANSS total & $77.6 \pm 19.0$ & $84.5 \pm 16.7$ & $74.4 \pm 19.6$ & $98.1 \pm 12.6$ & $79.7 \pm 9.8$ & $72.6 \pm 9.8$ & $55.0 \pm 9.6$ \\
\hline CDSS & $13.2 \pm 4.8$ & $13.2 \pm 5.0$ & $13.2 \pm 4.7$ & $14.1 \pm 5.5$ & $13.0 \pm 4.8$ & $13.7 \pm 4.7$ & $11.6 \pm 3.3$ \\
\hline PSP & $48.9 \pm 15.2$ & $45.5 \pm 15.6$ & $50.5 \pm 14.8$ & $45.0 \pm 13.2$ & $44.9 \pm 15.3$ & $47.6 \pm 14.1$ & $59.2 \pm 14.4$ \\
\hline Recreational drug use & $48.9 \%$ & $48.0 \%$ & $49.3 \%$ & $39.2 \%$ & $38.7 \%$ & $53.8 \%$ & $64.4 \%$ \\
\hline Seropositivity to T. gondii & $23.7 \%$ & $30.2 \%$ & $20.8 \%$ & $30.9 \%$ & $23.7 \%$ & $17.4 \%$ & $22.4 \%$ \\
\hline Seropositivity to CMV & $56.1 \%$ & $56.3 \%$ & $56.0 \%$ & $60.6 \%$ & $45.8 \%$ & $60.9 \%$ & $52.2 \%$ \\
\hline Seropositivity to HSV-1 & $58.0 \%$ & $56.3 \%$ & $58.8 \%$ & $63.8 \%$ & $44.1 \%$ & $64.1 \%$ & $53.7 \%$ \\
\hline Remitters & $68.60 \%$ & $0.00 \%$ & $100.00 \%$ & $57.70 \%$ & $54.80 \%$ & $61.30 \%$ & $90.40 \%$ \\
\hline
\end{tabular}

The total number of patients, proportion of males, age (mean \pm SEM), BMI (mean \pm SEM), waist circumference (mean \pm SEM) and clinical scores (mean \pm SEM) before treatment are indicated in the study sample as a whole (all patients, non-remitters and remitters), and in the indicated subtypes. The proportion (\%) of patients reporting recreational drug use, test seropositive for the indicated pathogens, or identified as remitters after 4 weeks of treatment with amisulpride are also indicated $B M I$ bone marrow index, PANSS Positive and Negative Syndrome Scale, PPANSS positive PANSS, NPANSS negative PANSS, GPANSS general psychopathology PANSS, CDSS Calgary Depression Scale for Schizophrenia, PSP Personal and Social Performance Scale, CMV cytomegalovirus, HSV-1 herpes simplex virus type 1

were not included in downstream analyses (Supplementary Table 1). In an exploratory analysis, we compared the levels of the 35 remaining proteins in remitters and nonremitters using univariate analysis. After correction for multiple test, none of these 35 proteins was present at different levels in remitters and non-remitters (Supplementary Table 2).

In contrast to univariate methods that assess the differential expression of proteins on a single feature level, multivariate classification methods such as regularized logistic regression allows for establishing a prediction model based on samples with known class outcomes, e.g., remission versus non-remission ${ }^{40}$. A set of clinical and biological variables with the best joint discriminatory ability to differentiate between classes could be identified, and the resulting prediction model could then be used to predict the class outcomes of new patient samples. As a second attempt to predict remission, we investigated the association between serum protein levels and remission using regularized logistic regression after adjustment for age, gender, body mass index (BMI), waist circumference, use of recreational drugs and seropositivity to $T$. gondii, CMV and HSV-1. Applying this method to the dataset did not allow for identifying proteins whose serum levels were associated with increased odds of being non-remitters (not shown).

One obvious explanation for this negative result could be that none of the studied serum proteins is relevant for discriminating remitters and non-remitters among FEP patients. Alternatively, the heterogeneity of psychotic disorders in terms of symptomatology and likely etiology and pathophysiology may impede the identification of underlying remission predictors in a general population of FEP patients. To overcome this issue, we sought to stratify FEP patients based on their individual symptomatology assessed using the PANSS instrument ${ }^{41}$.

\section{Patient clustering}

We sought to stratify patients in clusters in which patients within one cluster would be more similar (cohesion) than patients in the others (separation). We applied a two-step hierarchical unsupervised clustering method to a dataset consisting of the 30 individual PANSS scores of the 325 patients in the OPTiMiSE study sample, therefore resulting in four clusters: $\mathrm{C} 1 \mathrm{~A}$ and $\mathrm{C} 1 \mathrm{~B}$, and $\mathrm{C} 2 \mathrm{~A}$ and $\mathrm{C} 2 \mathrm{~B}$. We compared two methods for data 


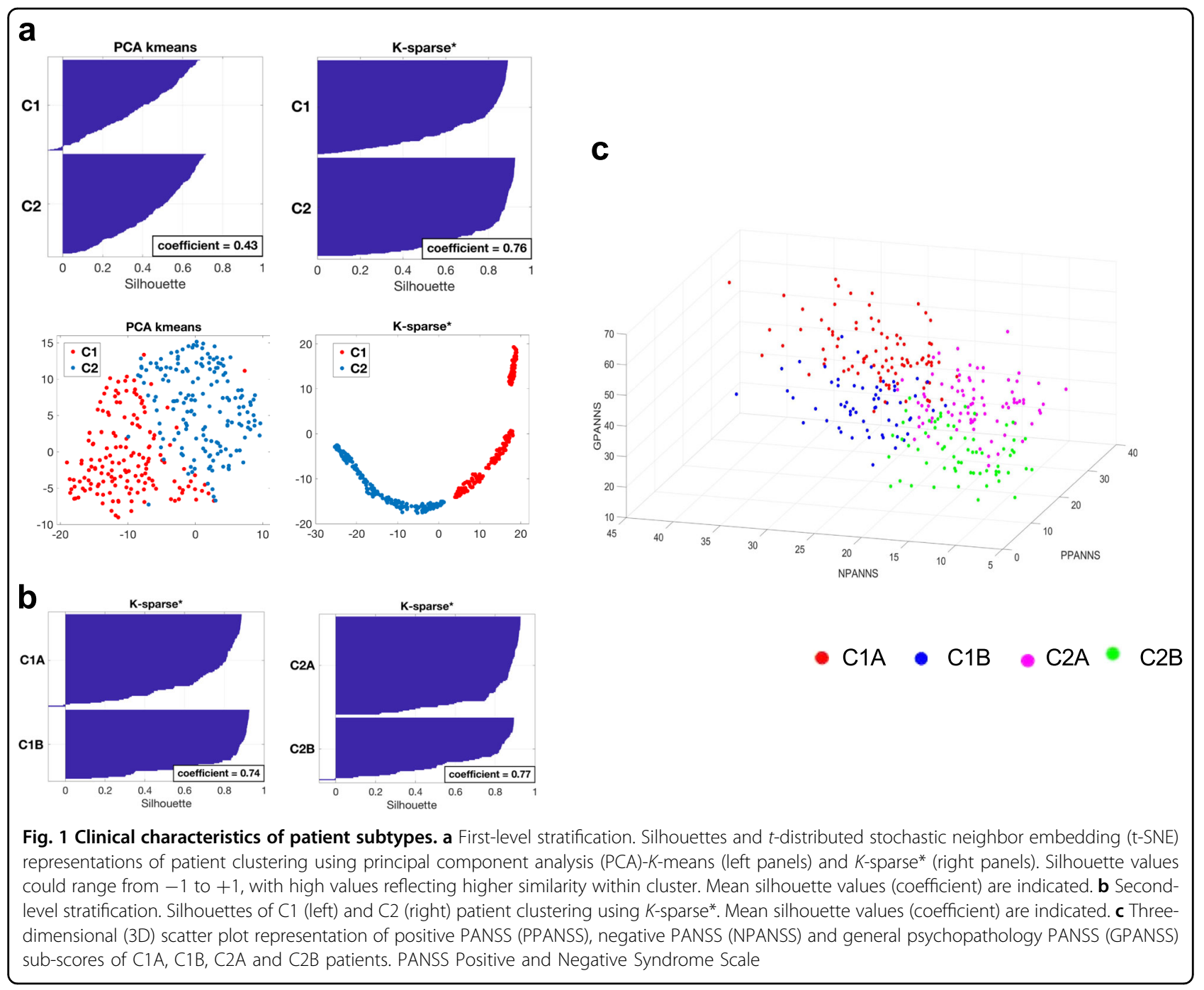

clustering: principal component analysis (PCA)-Kmeans ${ }^{42}$ that is a popular method for cluster analysis, and $K$-sparse* that is a modified version of $K$-sparse ${ }^{38}$. While both methods were successful at stratifying the 325 patients of the OPTiMiSE study sample, $K$-sparse* outperformed PCA- $K$-means as demonstrated by both a higher mean silhouette value (0.76 compared to 0.43 ) and $t$-distributed stochastic neighbor embedding ( $\mathrm{t}$-SNE) graphical representations (Fig. 1a). We therefore selected $K$-sparse* for data clustering. First-level classification using $K$-sparse* identified two subtypes: $\mathrm{C} 1(n=159)$ and C2 $(n=166)$. K-sparse* selected nine items that discriminated $\mathrm{C} 1$ and $\mathrm{C} 2$ patients, among which five belonged to the negative PANSS sub-scale (NPANSS) and four to the general psychopathology PANSS sub-scale (GPANSS) (Supplementary Table 3). Second-level classification identified four subtypes: C1A $(n=97)$ and C1B $(n=62)$ on one hand, and C2A $(n=95)$ and C2B $(n=71)$ on the other. $K$-sparse* selected eight PANSS items for discriminating $\mathrm{C} 1 \mathrm{~A}$ from $\mathrm{C} 1 \mathrm{~B}$ patients, among which four belonged to the positive PANSS (PPANSS) sub-scale and four to the GPANSS sub-scale (Supplementary Table 3). $K$-sparse* selected seven PANSS items for discriminating $\mathrm{C} 2 \mathrm{~A}$ from $\mathrm{C} 2 \mathrm{~B}$ patients, among which three belonged to the PPANSS sub-scale and four to the GPANSS sub-scale (Supplementary Table 3). In agreement with the nature and the weight of the PANSS items selected by $K$-sparse*, $\mathrm{C} 1 \mathrm{~A}$ and $\mathrm{C} 1 \mathrm{~B}$ patients exhibited more severe negative and general psychopathology symptoms compared to $\mathrm{C} 2 \mathrm{~A}$ and $\mathrm{C} 2 \mathrm{~B}$ patients respectively (Table 1 ). $\mathrm{C} 1 \mathrm{~A}$ and $\mathrm{C} 2 \mathrm{~A}$ patients exhibited more prominent positive and general psychopathology symptoms compared to $\mathrm{C} 1 \mathrm{~B}$ and $\mathrm{C} 2 \mathrm{~B}$ patients respectively (Table 1 ). Compared to other patients from the study sample, those from the C1A subtype exhibited more severe symptoms in the positive, negative and general psychopathology dimensions (Table 2, Fig. 1c). C1A patients also exhibited higher clinical scores as measured by the CGI and the CDSS and showed 


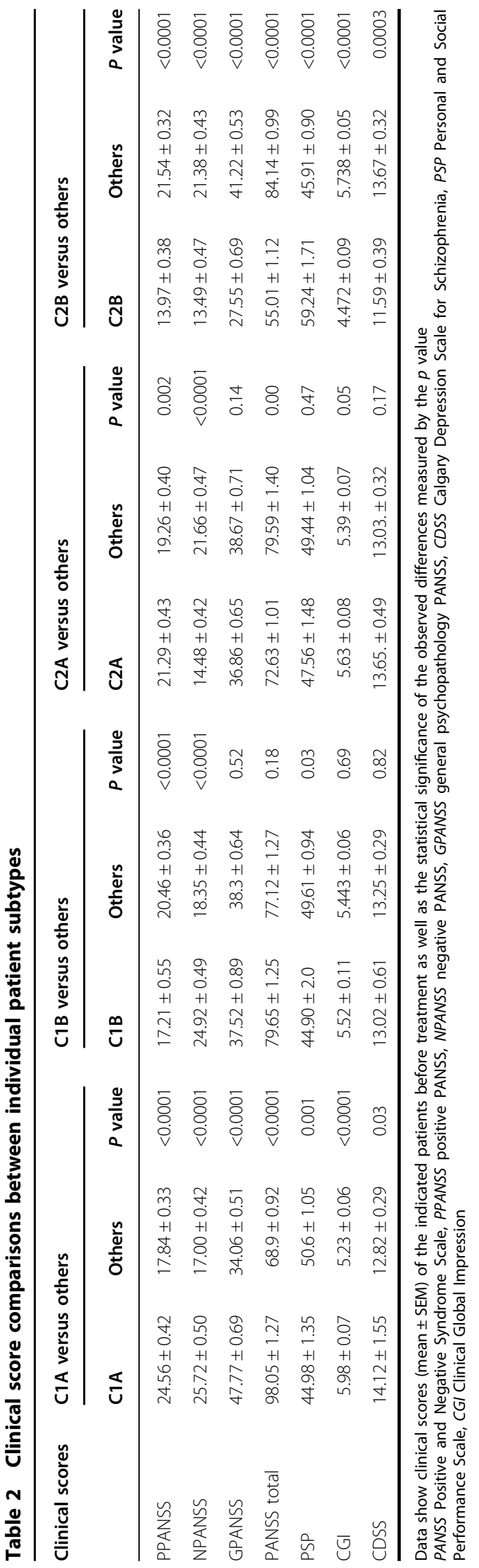

the worst psychosocial performance/functioning as measured by PSP scale (Table 2). In contrast, C2B patients exhibited less severe symptoms in the positive, negative and general psychopathology dimensions, exhibited lower CGI and CDSS scores and showed the best psychosocial performance/functioning as measured by the PSP scale (Table 2, Fig. 1c).

In summary, applying a two-step hierarchical unsupervised classification method to FEP patients identified four patient subtypes characterized by different symptom profiles. C1A patients exhibited the most severe symptoms in all dimensions, and $57.70 \%$ of them were remitters after 4-week treatment with amisulpride, compared to $68.6 \%$ in the study sample as a whole (Table 1). In contrast, $\mathrm{C} 2 \mathrm{~B}$ patients exhibited less severe symptoms, and $90.4 \%$ of them were remitters after 4 weeks of treatment (Table 1).

\section{Validation of the clustering solution}

The complexity of deriving clustering solutions makes validation crucial not only to ensure reproducibility but also to confirm that the derived clusters index clinically meaningful variations ${ }^{43,44}$. We first sought to validate our clustering solution using cross-validation, i.e., by first splitting data in a training and a test sample, and then assigning each patient of the test sample to one of the clusters derived from the training sample. Results from 50 independent random drawings showed that our clustering solution was robust with $86.8 \%$ to $95.5 \%$ of the patients (depending of the cluster) in the test sample being correctly classified (Supplementary Table 4).

As an alternative and complementary approach, we sought to validate our clustering solution on external biological measures, i.e., to investigate whether reducing clinical heterogeneity also reduces biological heterogeneity $^{45-47}$. To this aim, we searched for serum biomarkers that were present at different levels between clusters. Univariate analysis did not identify serum proteins that distinguished $\mathrm{C} 1 \mathrm{~B}$ or $\mathrm{C} 2 \mathrm{~A}$ patients from the others. In contrast, $\mathrm{C} 1 \mathrm{~A}$ patients exhibited statistically higher levels of IL-7, IL-15, IL-17, IFN- $\gamma$, TNF- $\alpha$, sICAM1 and sVCAM-1 after correction for multiple test (Table 3). The probability that seven biomarkers or more would have been expressed at statistically higher levels in 97 randomly selected patients (to match the number of $\mathrm{C} 1 \mathrm{~A}$ patients) compared to the others was $5.47 \times 10^{-6}$ as estimated by 10,000 successive random drawings (Supplementary Table 5). We also found that $\mathrm{C} 2 \mathrm{~B}$ patients exhibited lower levels of CXCL12 and higher levels of IL8. Effect sizes were small to medium $(0.5>$ Cohen's $d$ coefficient $>0.2$ ) for IFN- $\gamma$, IL-7, IL-17, TNF- $\alpha$, sICAM-1, CXCL12 and sVCAM-1, and medium to high (1.0> Cohen's $d$ coefficient $>0.5$ ) for both IL-15 and IL-8. Because the $K$-sparse* clustering approach that we have 


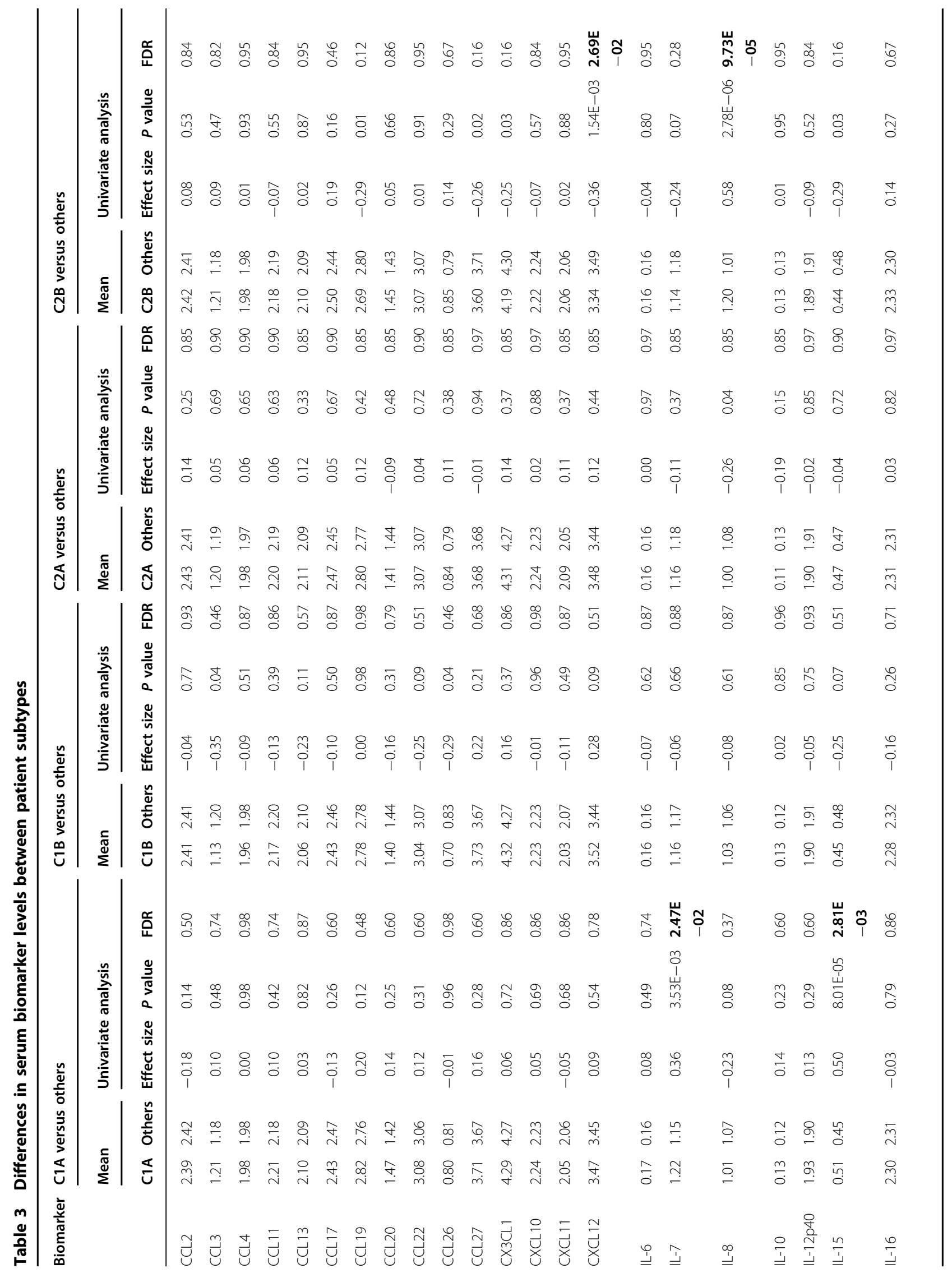




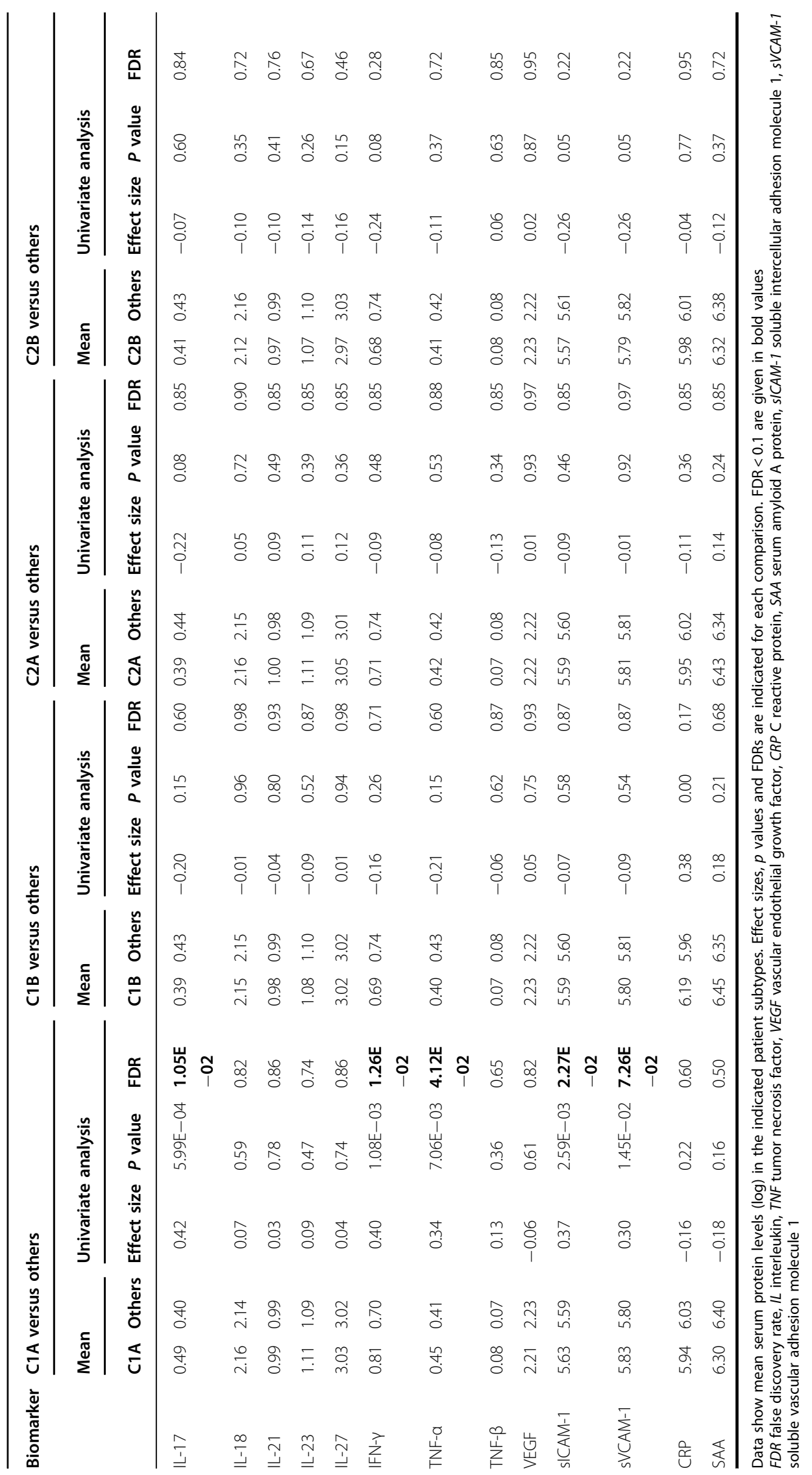


used to define $\mathrm{C} 1 \mathrm{~A}, \mathrm{C} 1 \mathrm{~B}, \mathrm{C} 2 \mathrm{~A}$ and $\mathrm{C} 2 \mathrm{~B}$ subtypes was based on clinical features only, the fact that several peripheral biomarkers distinguished at least two patient subtypes from the others validated our clustering approach on external biological measures ${ }^{48}$.

\section{Predicting remission in individual patient subtypes}

As an attempt to identify serum biomarkers associated with remission in individual patient subtypes, we applied regularized logistic regression to clinical and biological data from C1A, C1B, C2A and C2B patients. None of the analyzed variables was associated with remission in $\mathrm{C} 1 \mathrm{~B}$, $\mathrm{C} 2 \mathrm{~A}$ and $\mathrm{C} 2 \mathrm{~B}$ patients (not shown). In striking contrast, lower serum levels of IL-15, higher serum levels of CXCL12, seropositivity to CMV, use of recreational drugs and being younger were all associated with increased odds of being non-remitters in C1A patients (Table 4, model 1). Among these five variables, IL-15 was selected in $99.6 \%$ of the training/test runs and had a $p$ value $<0.001$. To estimate the predictive value of these five combined variables, we applied a regularized logistic regression to these five variables only (Table 4 , model 2 ). All variables were selected more than $95 \%$ of the time and exhibited $p$ values $<0.1$. The predictive value of this model, assessed by the ROC curve was $73 \pm 0.10 \%$, and its specificity and selectivity were $45 \pm 0.09 \%$ and $83 \pm 0.03 \%$, respectively.

\section{Discussion}

Heterogeneity of patients with mental disorders may impede identification of adequate predictors of remis$\operatorname{sion}^{43}$. In keeping with this hypothesis, we have failed to identify serum biomarkers associated with remission in non-stratified FEP patients. To overcome this problem, we used a hierarchical clustering approach to identify subtypes of patients based on their clinical symptoms. Several unsupervised clustering methods have been used to stratify patients with mental disorders based on clinical symptoms and case history variables ${ }^{49-53}$ or social cognitive measures ${ }^{54}$. Given a set of data points, clustering methods aim to partition data into a specified number $(k)$ of clusters, such that the samples in each cluster are more similar to one another than to those in the other clusters. This entails defining a measure of similarity or distance between data points. As recently pointed out ${ }^{43}$, the outcome of clustering is highly dependent on the input data with relatively little convergence towards a coherent and consistent set of subtypes. Unfortunately, the biological relevance of the few subtypes identified so far was generally limited and did not clearly reflect underlying biological mechanisms. Here, we have used a two-step hierarchical unsupervised clustering method to stratify FEP patients into four subtypes, termed C1A, C1B, C2A and $\mathrm{C} 2 \mathrm{~B}$, based on their clinical symptoms. $\mathrm{C} 1 \mathrm{~A}$ patients were characterized by the most severe symptoms in the positive, negative and general physiopathology dimensions. In contrast, $\mathrm{C} 2 \mathrm{~B}$ patients were the least severely affected. Most importantly, C1A and C2B patients did not only differ from other patients in terms of symptoms severity but also exhibited specific peripheral immune signatures suggesting that these subtypes reflected distinct pathophysiological entities ${ }^{45,55}$. Our study therefore provides the proof of concept that clustering methods aimed at reducing clinical heterogeneity may also reduce biological heterogeneity.

Several authors in the field have tried to stratify psychosis spectrum patients on the basis of symptoms. In a pioneer study, Dollfus et al $^{51}$ have used the Ward's method of hierarchical clustering to identify four subtypes of schizophrenia patients that they called "positive", "negative", "mixed" and "disorganized". These four subtypes are very similar to the four subsets that we describe here, with our $\mathrm{C} 1 \mathrm{~B}, \mathrm{C} 2 \mathrm{~A}, \mathrm{C} 1 \mathrm{~A}$ and $\mathrm{C} 2 \mathrm{~B}$ subsets being very similar to Dollfus' "positive", "negative", "mixed" and "disorganized" subtypes, respectively.

In contrast to the current "one size fits all" or "trial and error" approach in healthcare, stratified medicine aims at sorting a population into biologically relevant subtypes. We found that the vast majority (90.4\%) of C2B patients were remitters after treatment. This agrees with previous studies which have shown that patients with less severe negative symptoms are more likely to be remitters than others ${ }^{9}$. In contrast, the proportion of remitters among $\mathrm{C} 1 \mathrm{~A}, \mathrm{C} 1 \mathrm{~B}$ and C2B patients ranged between $54.8 \%$ and $61.3 \%$. Thus, the clustering solution that we describe here constitutes a first step towards stratified medicine for psychotic patients.

In support of a critical role of inflammation in psychiatric diseases, add-on treatments with antiinflammatory drugs have been tested in severe and treatment-resistant psychiatric patients ${ }^{56-64}$. For example, acetyl salicylic acid (Aspirin) which interrupts the immuno-inflammatory cascade by inhibiting cyclooxygenase (COX)-1 and COX-2 showed promising results as an add-on treatment of schizophrenia in comparison to treatment as usual ${ }^{60,63}$. In most cases however, add-on anti-inflammatory treatments in psychotic patients only provided modest improvements in clinical outcome. This could be explained if only a subtype of the treated patients exhibited a pro-inflammatory profile at baseline. In agreement with this latter hypothesis, an add-on trial in patients with psychotic disorders showed that those with increased CRP levels had the largest response to add-on Aspirin as compared to those with lower levels ${ }^{65}$. Compared to others, C1A patients exhibited higher levels of IL7, IL-15, IL-17, IFN- $\gamma$, TNF- $\alpha$, sICAM-1 and SVCAM-1. Therefore, a reasonable and testable hypothesis is that C1A patients would be those that could benefit the most from add-on anti-inflammatory treatment. On another topic, several authors have proposed that inflammation 
Table 4 Clinical and biological variables associated with non-remission in C1A patients

\begin{tabular}{|c|c|c|c|c|c|c|}
\hline \multirow[t]{2}{*}{ Variable } & \multicolumn{3}{|l|}{ Model 1} & \multicolumn{3}{|l|}{ Model 2} \\
\hline & $\begin{array}{l}\text { Proportion of } \\
\text { selection }\end{array}$ & $\begin{array}{l}\text { Weighted mean odds } \\
\text { ratio } \pm \text { SD }\end{array}$ & $P$ value & $\begin{array}{l}\text { Proportion of } \\
\text { selection }\end{array}$ & $\begin{array}{l}\text { Weighted mean odds } \\
\text { ratio } \pm \text { SD }\end{array}$ & $P$ value \\
\hline Sex (being male) & 0.315 & $1.156 \pm 0.013$ & 0.572 & & & \\
\hline Age & 0.677 & $0.817 \pm 0.008$ & 0.099 & 0.953 & $0.804 \pm 0.006$ & 0.095 \\
\hline BMI & 0.312 & $1.077 \pm 0.004$ & 0.654 & & & \\
\hline Waist circumference & 0.341 & $1.068 \pm 0.002$ & 0.518 & & & \\
\hline Recreational drug use & 0.774 & $1.240 \pm 0.019$ & 0.070 & 0.967 & $1.393 \pm 0.016$ & 0.048 \\
\hline Seropositivity to HSV-1 & 0.225 & $1.130 \pm 0.012$ & 0.757 & & & \\
\hline Seropositivity to CMV & 0.702 & $1.283 \pm 0.021$ & 0.093 & 0.963 & $1.490 \pm 0.028$ & 0.012 \\
\hline $\begin{array}{l}\text { Seropositivity to } \\
\text { Toxoplasma }\end{array}$ & 0.710 & $1.266 \pm 0.025$ & 0.119 & & & \\
\hline CCL2 & 0.168 & $1.029 \pm 0.001$ & 0.693 & & & \\
\hline CCL3 & 0.325 & $0.912 \pm 0.004$ & 0.690 & & & \\
\hline CCL4 & 0.162 & $1.012 \pm 0.001$ & 0.764 & & & \\
\hline CCL11 & 0.244 & $1.057 \pm 0.003$ & 0.643 & & & \\
\hline CCL13 & 0.148 & $1.036 \pm 0.004$ & 0.684 & & & \\
\hline CCL17 & 0.179 & $1.024 \pm 0.009$ & 0.517 & & & \\
\hline CCL19 & 0.359 & $0.976 \pm 0.006$ & 0.640 & & & \\
\hline CCL20 & 0.236 & $0.982 \pm 0.001$ & 0.763 & & & \\
\hline CCL22 & 0.151 & $0.971 \pm 0.004$ & 0.668 & & & \\
\hline CCL26 & 0.564 & $1.163 \pm 0.010$ & 0.291 & & & \\
\hline CCL27 & 0.154 & $1.048 \pm 0.002$ & 0.626 & & & \\
\hline CX3CL1 & 0.168 & $0.962 \pm 0.002$ & 0.784 & & & \\
\hline CXCL10 & 0.155 & $1.031 \pm 0.004$ & 0.754 & & & \\
\hline CXCL11 & 0.321 & $0.937 \pm 0.002$ & 0.634 & & & \\
\hline CXCL12 & 0.715 & $1.240 \pm 0.019$ & 0.077 & 0.964 & $1.369 \pm 0.012$ & 0.020 \\
\hline IL-6 & 0.416 & $0.913 \pm 0.002$ & 0.656 & & & \\
\hline IL-7 & 0.183 & $0.950 \pm 0.002$ & 0.803 & & & \\
\hline IL-8 & 0.483 & $1.148 \pm 0.010$ & 0.528 & & & \\
\hline IL-10 & 0.372 & $1.093 \pm 0.004$ & 0.743 & & & \\
\hline IL-12p40 & 0.281 & $0.947 \pm 0.002$ & 0.591 & & & \\
\hline IL-13 & 0.284 & $0.953 \pm 0.001$ & 0.717 & & & \\
\hline IL-15 & 0.996 & $0.756 \pm 0.009$ & $5.00 \mathrm{E}-04$ & 1.000 & $0.585 \pm 0.005$ & $\begin{array}{l}4.00 \mathrm{E} \\
-04\end{array}$ \\
\hline IL-16 & 0.288 & $0.933 \pm 0.002$ & 0.711 & & & \\
\hline |L-17 & 0.405 & $0.941 \pm 0.002$ & 0.520 & & & \\
\hline |L-18 & 0.18 & $1.000 \pm 0.003$ & 0.640 & & & \\
\hline $\mid \mathrm{L}-21$ & 0.211 & $0.962 \pm 0.002$ & 0.812 & & & \\
\hline IL-23 & 0.256 & $0.957 \pm 0.002$ & 0.709 & & & \\
\hline IL-27 & 0.238 & $1.067 \pm 0.004$ & 0.796 & & & \\
\hline IFN- $\gamma$ & 0.21 & $0.954 \pm 0.002$ & 0.689 & & & \\
\hline TNF- $a$ & 0.553 & $1.226 \pm 0.016$ & 0.240 & & & \\
\hline TNF- $\beta$ & 0.32 & $0.946 \pm 0.001$ & 0.664 & & & \\
\hline VEGF & 0.134 & $1.025 \pm 0.002$ & 0.596 & & & \\
\hline sICAM-1 & 0.266 & $0.924 \pm 0.003$ & 0.669 & & & \\
\hline sVCAM-1 & 0.474 & $0.897 \pm 0.003$ & 0.388 & & & \\
\hline CRP & 0.67 & $0.841 \pm 0.005$ & 0.186 & & & \\
\hline SAA & 0.088 & $0.973 \pm 0.000$ & 0.233 & & & \\
\hline
\end{tabular}

Data show the variables that were included in regularized regression logistic models. All variables listed in the left column were included in model 1 . Recreational drug use, seropositivity to CMV, IL-15, CXCL12 and age were included in model 2. For each variable, the proportion of drawings (out of 2000) in which the variable was selected is indicated, as well as the weighted mean odds ratio (ORs) \pm SD. The $p$ values were computed adjusting a single elastic net model with the mean $a$ and mean $\lambda$ as hyper-parameters. In model 1 , mean $a$ and mean $\lambda$ hyper-parameters were $0.37 \pm 0.29$ and $0.26 \pm 0.28$ respectively. Mean area under the curve (AUC) was $0.62 \pm$ 0.09 with minimal and maximal values of 0.42 and 0.82 respectively. In Model 2, mean $a$ and mean $\lambda$ hyper-parameters were $0.11 \pm 0.07$ and $0.13 \pm 0.13$ respectively. Mean AUC was $0.73 \pm 0.1$ with minimal and maximal values of 0.36 and 0.95 respectively. The $p$ values $<0.1$ are given in bold values

$B M I$ bone marrow index, CMV cytomegalovirus, HSV-1 herpes simplex virus type 1, IL interleukin, TNF tumor necrosis factor, VEGF vascular endothelial growth factor, CRP C reactive protein, SAA serum amyloid A protein, sICAM-1 soluble intercellular adhesion molecule 1, sVCAM-1 soluble vascular adhesion molecule 1 
was associated with poor clinical outcome in psychosis ${ }^{66-69}$. In agreement with these studies, C1A patients were both characterized by higher levels of several inflammatory biomarkers and a lower proportion of non-remitters (57.7\% compared to $68.6 \%$ in the study sample as a whole, and $90.4 \%$ in the C2B subtype).

Compared to stratified medicine, personalized medicine builds on a finer sub-classification of patients to enable individual tailoring of treatment to maximize response. Bearing this in mind, we have used regularized logistic regression to select variables that could predict remission in individual patient subtypes. In C1A patients but not in others, lower levels of IL-15, higher levels of CXCL12, recreational drug use, being seropositive to CMV and being younger were all associated with increased odds of being non-remitters after adjustment for covariates suspected to impact cytokine levels or response to treatment. While IL-15 is mainly known for its role in regulating natural killer and $\mathrm{T}$ cells ${ }^{70,71}$, it is also produced by astrocytes and neural progenitors ${ }^{72,73}$, regulates neurogenesis and exerts anti-depressive effects in mice ${ }^{74,75}$. Likewise, while CXCL12 was first described as a chemotactic factor for lymphocytes and macrophages, it is also secreted by glial cells and neurons and plays a role in brain plasticity and function ${ }^{76}$. One of the two CXCL12 receptors, CXCR4, acts at both the synaptic and postsynaptic levels by promoting the release of glutamate and $\gamma$-aminobutyric acid (GABA) and by activating the voltage-gated $\mathrm{K}$ channel Kv2.1, respectively. Whether and how IL-15 and CXCL12 impact response to antipsychotics remains to be elucidated.

In addition to lower levels of IL-15 and higher levels of CXCL12, being seropositive to CMV and the use of recreational drugs were both associated with an increased risk of being non-remitters in $\mathrm{C} 1 \mathrm{~A}$ patients. Previous studies have identified CMV infection ${ }^{77}$ and use of recreational drugs ${ }^{78}$ as risk factors for schizophrenia. Why these two variables are also associated with an increased risk of being non-remitters in C1A patients is unclear.

Our results, if replicated, could pave the way for the development of a blood-based assisted clinical decision support system for selecting the most appropriate treatment in psychotic patients.

\section{Acknowledgements}

We thank all the patients who participated in the OPTiMiSE study. The OPTiMiSE study was funded by the European Commission within the 7th Program (HEALTH-F2- 2010-242114). This work was also supported by the Investissements d'Avenir program managed by the Agence Nationale pour la Recherche (ANR) under reference ANR-11-IDEX-0004-02 (Labex BioPsy) (to W.B. H.A.). Additional financial supports were obtained from the Fondation de France (to N.G.) and the Fondation FondaMental (to N.G.). Additional supports were obtained by the LABEX SIGNALIFE (\#ANR-11-LABX-0028-01) and the FHU OncoAge. The Leboyer's team is affiliated with the Paris School of Neuroscience (ENP) and the BioPsy Laboratory of Excellence. Members of the OPTiMiSE study group are R.S. Kahn, I. Winter van Rossum, S. Leucht, P. McGuire, S.W. Lewis, M. Leboyer, C. Arango, P. Dazzan, R. Drake, S. Heres, C. M.
Diaz- Caneja, D. Rujescu, M. Weiser, S. Galderisi, B. Glenthoj, M.J.C. Eijkemans, W. W. Fleischhacker, S. Kapur, and I.E. Sommer. The authors would like to acknowledge this list of collaborators: Kahn RS, Sommer IE, Winter-van Rossum I, Somers M, Ywema PC, Kapur S, McGuire P, Leboyer M, Meyer-Lindenberg A, Lewis SW, Leucht S, Arango C, Fleischhacker WW, Meijering AL, Petter J, Van de Brug R, Schotsman J, Zwerver J, Peuskens J, De Hert M, Thys E, Hranov LG, Hranov V, Libiger J, Köhler R, Mohr P, Glenthoj B, Broberg B, Düring S, Baandrup L, Jamain S, Heres S, Rujescu D, Giegling I, Weiser M, Bar Heim M, Davidson M, Galderisi S, Bucci P, Mucci A, Rybakowski J, Remlinger-Molenda A, Gonen I, Radu P, Díaz-Marsá M, Rodriguez A, Palomo T, Rodriguez-Jimenez R, GarcíaPortilla P, Bernardo M, Bobes J, Vilares Oliveira C, Berger G, Wildt C, Dazzan P, Perez-Iglesias R, Drake R, Gregory S, Wilson D, Díaz-Caneja CM, Eijkemans MJC.

\section{Code availability}

The code for K-sparse* unsupervised classification will be made available upon request.

\section{Author details}

'Université Côte d'Azur, Centre National de la Recherche Scientifique, Institut de Pharmacologie Moléculaire et Cellulaire, Valbonne, France. ${ }^{2}$ Université Paris Est Créteil, Faculté de Medicine Institut, National de la Santé et de la Recherche Médicale, Créteil, France. ${ }^{3}$ Université Côte d'Azur, Centre National de la Recherche Scientifique, Laboratoire Informatique Signaux et Systèmes de Sophia Antipolis, Sophia Antipolis, France. ${ }^{4}$ Assistance Publique Hôpitaux de Marseille, Marseille, France. ${ }^{5}$ Department of Neuroscience and Department of Psychiatry, University Medical Center Groningen, Rijks Universiteit Groningen, Groningen, The Netherlands. ${ }^{6}$ Department of Medical and Biological Psychology, University of Bergen, Bergen, Norway. ${ }^{7}$ Department of Psychiatry and Psychotherapy, Technische Universität München, München, Germany. ${ }^{8}$ Department of Psychosis Studies, Institute of Psychiatry, National Institute for Health Research, Mental Health Biomedical Research Centre, King's College London, London, UK. ${ }^{9}$ Child and Adolescent Psychiatry Department, Hospital General Universitario Gregorio Marañón, Universidad Complutense, Madrid, Spain. ${ }^{10}$ Department of Psychiatry, Psychotherapy and Psychosomatic Medicine, Medical University Innsbruck, Innsbruck, Austria. ${ }^{11}$ Department of Psychiatry, University of Halle, Halle, Germany. ${ }^{12}$ Faculty of Health and Medical Sciences, Center for Neuropsychiatric Schizophrenia Research and Center for Clinical Intervention and Neuropsychiatric Schizophrenia Research, Psychiatric Hospital Center Glostrup, University of Copenhagen, Copenhagen, Denmark.

${ }^{13}$ Department of Psychiatry, Brain Center Rudolf Magnus, UMC Utrecht, Utrecht, The Netherlands. ${ }^{14}$ John Hopkins School of Medicine, The John Hopkins Hospital, Baltimore, USA. ${ }^{15}$ Division of Psychology and Mental Health, School of Health Sciences, Faculty of Biology, Medicine and Health, Manchester Academic. Health Sciences Centre (MAHSC), University of Manchester, Manchester, UK. ${ }^{16}$ Assistance Publique Hôpitaux de Paris, Pole de Psychiatrie et Addictologie, Hopitaux Universitaires Henri Mondor, Créteil, France. ${ }^{17}$ Fondation Fondamental, Hôpital Albert Chenevier Pôle de Psychiatrie, Créteil, France

\section{Conflict of interest}

The authors declare that they have no conflict of interest.

\section{Publisher's note}

Springer Nature remains neutral with regard to jurisdictional claims in published maps and institutional affiliations.

Supplementary Information accompanies this paper at (https://doi.org/ 10.1038/s41398-018-0366-5).

Received: 27 November 2018 Accepted: 10 December 2018 Published online: 17 January 2019

\section{References}

1. American Psychiatric Association. Diagnostic and Statistical Manual of Mental Disorders (DSM-5 $\left.{ }^{\circledR}\right)$, 5th edn (American Psychiatric Publishing, Arlington, 2013).

2. Kane, J., Leucht, S., Carpenter, D. \& Docherty, J., Disorders, E.C.P.f.O.P.T.o.P. The expert consensus guideline series. Optimizing pharmacologic treatment of 
psychotic disorders. Introduction: methods, commentary, and summary. J. Clin. Psychiatry 64 (Suppl 12), 5-19 (2003).

3. Leucht, S., Arbter, D., Engel, R. R., Kissling, W. \& Davis, J. M. How effective are second-generation antipsychotic drugs? A meta-analysis of placebocontrolled trials. Mol. Psychiatry 14, 429-447 (2009).

4. Lieberman, J. A., Dixon, L. B. \& Goldman, H. H. Early detection and intervention in schizophrenia: a new therapeutic model. JAMA 310, 689-690 (2013).

5. Dixon, L. B. \& Stroup, T. S. Medications for first-episode psychosis: making a good start. Am. J. Psychiatry 172, 209-211 (2015).

6. Fond, G. et al. The promise of biological markers for treatment response in first- episode psychosis: a systematic review. Schizophr. Bull. 41, 559-573 (2015).

7. Leucht, S. \& Kane, J. M. Measurement-based psychiatry: definitions of response, remission, stability, and relapse in schizophrenia. J. Clin. Psychiatry 67, 1813-1814 (2006).

8. Andreasen, N. C. et al. Remission in schizophrenia: proposed criteria and rationale for consensus. Am. J. Psychiatry 162, 441-449 (2005).

9. Jager, $M$. et al. Prediction of symptom remission in schizophrenia during inpatient treatment. World J. Biol. Psychiatry 10, 426-434 (2009).

10. Parellada, M. et al. Predictors of functional and clinical outcome in early-onset first-episode psychosis: the child and adolescent first episode of psychosis (CAFEPS) study. J. Clin. Psychiatry 76, e1441-e1448 (2015).

11. Dean, K. \& Murray, R. M. Environmental risk factors for psychosis. Dialog. Clin. Neurosci. 7, 69-80 (2005).

12. Eaton, W. W. et al. Association of schizophrenia and autoimmune diseases: linkage of Danish national registers. Am. J. Psychiatry 163, 521-528 (2006).

13. Yolken, R. H. \& Torrey, E. F. Are some cases of psychosis caused by microbial agents? A review of the evidence. Mol. Psychiatry 13, 470-479 (2008).

14. Leweke, F. M. et al. Antibodies to infectious agents in individuals with recent onset schizophrenia. Eur. Arch. Psychiatry Clin. Neurosci. 254, 4-8 (2004).

15. Niebuhr, D. W., Millikan, A. M., Yolken, R., Li, Y. \& Weber, N. S. Results from a hypothesis generating case-control study: herpes family viruses and schizophrenia among military personnel. Schizophr. Bull. 34, 1182-1188 (2008).

16. Potvin, S. et al. Inflammatory cytokine alterations in schizophrenia: a systematic quantitative review. Biol. Psychiatry 63, 801-808 (2008).

17. Miller, B. J., Buckley, P., Seabolt, W., Mellor, A. \& Kirkpatrick, B. Meta-analysis of cytokine alterations in schizophrenia: clinical status and antipsychotic effects. Biol. Psychiatry 70, 663-671 (2011).

18. Upthegrove, R., Manzanares-Teson, N. \& Barnes, N. M. Cytokine function in medication-naive first episode psychosis: a systematic review and metaanalysis. Schizophr. Res. 155, 101-108 (2014).

19. Crespo-Facorro, B. et al. Long-term (3-year) effectiveness of haloperidol, risperidone and olanzapine: results of a randomized, flexible-dose, open-labe comparison in first-episode nonaffective psychosis. Psychopharmacology (Berl.) 219, 225-233 (2012)

20. Kubistova, A., Horacek, J. \& Novak, T. Increased interleukin-6 and tumor necrosis factor alpha in first episode schizophrenia patients versus healthy controls. Psychiatr. Danub. 24 (Suppl. 1), S153-S156 (2012).

21. MacDowell, K. S. et al. Risperidone normalizes increased inflammatory parameters and restores anti-inflammatory pathways in a model of neuroinflammation. Int. J. Neuropsychopharmacol. 16, 121-135 (2013).

22. de Witte, L. et al. Cytokine alterations in first-episode schizophrenia patients before and after antipsychotic treatment. Schizophr. Res. 154, 23-29 (2014).

23. Ding, M. et al. Activation of Th17 cells in drug naive, first episode schizophrenia. Prog. Neuropsychopharmacol. Biol. Psychiatry 51, 78-82 (2014).

24. Leucht, S. et al. The optimization of treatment and management of schizophrenia in Europe (OPTiMiSE) trial: rationale for its methodology and a review of the effectiveness of switching antipsychotics. Schizophr. Bull. 41, 549-558 (2015).

25. Kahn, R. S. et al. Effectiveness of antipsychotic drugs in first-episode schizophrenia and schizophreniform disorder: an open randomised clinical trial. Lancet 371, 1085-1097 (2008).

26. Guy, W. Clinical Global Impression, ECDEU Assessment Manual for Psychopharmacology (National Institute of Mental Health, Rockville, 1976).

27. Addington, D., Addington, J. \& Schissel, B. A depression rating scale for schizophrenics. Schizophr. Res. 3, 247-251 (1990).

28. Morosini, P. L., Magliano, L., Brambilla, L., Ugolini, S. \& Pioli, R. Development, reliability and acceptability of a new version of the DSM-IV Social and Occupational Functioning Assessment Scale (SOFAS) to assess routine social functioning. Acta Psychiatr. Scand. 101, 323-329 (2000).
29. Buka, S. L. et al. Maternal infections and subsequent psychosis among offspring. Arch. Gen. Psychiatry 58, 1032-1037 (2001).

30. Zou, H. \& Hastie, T. Regularization and variable selection via the elastic net. J. $R$. Stat. Soc. B 67, 301-320 (2005).

31. Iniesta, R. et al. Combining clinical variables to optimize prediction of antidepressant treatment outcomes. J. Psychiatr. Res. 78, 94-102 (2016).

32. Fawcett, T. An introduction to ROC analysis. Pattern Recognit. Lett. 27, 861-874 (2006).

33. R Core Team. A Language and Environment for Statistical Computing. (R Foundation for Statistical Computing:, Vienna, 2016).

34. Kuhn, M. Caret package. J. Stat. Softw. 28, 1-26 (2008).

35. Friedman, J., Hastie, T. \& Tibshirani, R. Regularization paths for generalized linear models via coordinate descent. J. Stat. Softw. 33, 1-22 (2010).

36. Robin, $X$. et al. pROC: an open-source package for $\mathrm{R}$ and $\mathrm{S}+$ to analyze and compare ROC curves. BMC Bioinforma. 12, 77 (2011).

37. Candia, J. \& Tsang, J. S. eNetXplorer: an R package for the quantitative exploration of elastic net families for generalized linear models (April 30, 2018).

38. Gilet, C., Deprez, M., Caillau, J.-B. \& Barlaud, M. Clustering with feature selection using alternating minimization. Application to computational biology. Published 2017 in ArXiv.

39. Kahn, R. S. et al. Amisulpride and olanzapine followed by open-label treatment with clozapine in first-episode schizophrenia and schizophreniform disorder (OPTiMiSE): a three-phase switching study. Lancet Psychiatry 5, 797-807 (2018).

40. Preece R. L., Han, S. Y. S. \& Bahn, S. Proteomic approaches to identify bloodbased biomarkers for depression and bipolar disorders. Expert Rev. Proteomics 15, 325-340 (2018)

41. Kay, S. R., Fiszbein, A. \& Opler, L. A. The positive and negative syndrome scale (PANSS) for schizophrenia. Schizophr. Bull. 13, 261-276 (1987).

42. Iniesta, R., Stahl, D. \& McGuffin, P. Machine learning, statistical learning and the future of biological research in psychiatry. Psychol. Med. 46, 2455-2465 (2016).

43. Marquand, A. F., Wolfers, T., Mennes, M., Buitelaar, J. \& Beckmann, C. F. Beyond lumping and splitting: a review of computational approaches for stratifying psychiatric disorders. Biol. Psychiatry Cogn. Neurosci. Neuroimaging 1, 433-447 (2016).

44. Bzdok, D. \& Meyer-Lindenberg, A. Machine learning for precision psychiatry: opportunities and challenges. Biol. Psychiatry Cogn. Neurosci. Neuroimaging 3, 223-230 (2018)

45. Schumann, G. et al. Stratified medicine for mental disorders. Eur. Neuropsychopharmacol. 24, 5-50 (2014).

46. Chaste, P. et al. A genome-wide association study of autism using the Simons Simplex Collection: does reducing phenotypic heterogeneity in autism increase genetic homogeneity? Biol. Psychiatry 77, 775-784 (2015).

47. Insel, T. R. \& Cuthbert, B. N. Medicine. Brain disorders? Precisely. Science $\mathbf{3 4 8}$ 499-500 (2015).

48. Steckler, A. \& McLeroy, K. R. The importance of external validity. Am. J. Public Health 98, 9-10 (2008).

49. Farmer, A. E., McGuffin, P. \& Spitznagel, E. L. Heterogeneity in schizophrenia: a cluster-analytic approach. Psychiatry Res. 8, 1-12 (1983).

50. Castle, D. J., Sham, P. C., Wessely, S. \& Murray, R. M. The subtyping of schizophrenia in men and women: a latent class analysis. Psychol. Med. 24, 41-51 (1994).

51. Dollfus, S. et al. Identifying subtypes of schizophrenia by cluster analyses. Schizophr. Bull. 22, 545-555 (1996).

52. Kendler, K. S., Karkowski, L. M. \& Walsh, D. The structure of psychosis: latent class analysis of probands from the Roscommon Family Study. Arch. Gen. Psychiatry 55, 492-499 (1998).

53. Murray, $\vee$. et al. Dimensions and classes of psychosis in a population cohort: a four-class, four-dimension model of schizophrenia and affective psychoses. Psychol. Med. 35, 499-510 (2005).

54. Bell, M. D., Corbera, S., Johannesen, J. K., Fiszdon, J. M. \& Wexler, B. E. Social cognitive impairments and negative symptoms in schizophrenia: are there subtypes with distinct functional correlates? Schizophr. Bull. 39, 186-196 (2013).

55. Insel, $T$. et al. Research domain criteria (RDoC): toward a new classification framework for research on mental disorders. Am. J. Psychiatry 167, 748-751 (2010).

56. Laan, W. et al. Adjuvant aspirin therapy reduces symptoms of schizophrenia spectrum disorders: results from a randomized, double-blind, placebo- controlled trial. J. Clin. Psychiatry 71, 520-527 (2010).

57. Berk, M. et al. Aspirin: a review of its neurobiological properties and therapeutic potential for mental illness. BMC Med. 11, 74 (2013). 
58. Berthold-Losleben, M., Heitmann, S. \& Himmerich, H. Anti-inflammatory drugs in psychiatry. Inflamm. Allergy Drug. Targets 8, 266-276 (2009).

59. Sommer, I. E., de Witte, L., Begemann, M. \& Kahn, R. S. Nonsteroidal antiinflammatory drugs in schizophrenia: ready for practice or a good start? A meta- analysis. J. Clin. Psychiatry 73, 414-419 (2012).

60. Sommer, I. E. et al. Efficacy of anti-inflammatory agents to improve symptoms in patients with schizophrenia: an update. Schizophr. Bull. $\mathbf{4 0}$, 181-191 (2014).

61. Fond, G. et al. Effectiveness and tolerance of anti-inflammatory drugs' add-on therapy in major mental disorders: a systematic qualitative review. Acta Psychiatr. Scand. 129, 163-179 (2014).

62. Andrade, C. Nonsteroidal anti-inflammatory drugs and 5-HT(3) serotonin receptor antagonists as innovative antipsychotic augmentation treatments for schizophrenia. J. Clin. Psychiatry 75, e707-e709 (2014).

63. Nitta, M. et al. Adjunctive use of nonsteroidal anti-inflammatory drugs for schizophrenia: a meta-analytic investigation of randomized controlled trials. Schizophr. Bull. 39, 1230-1241 (2013).

64. Muller, N., Myint, A. M., Krause, D., Weidinger, E. \& Schwarz, M. J. Antiinflammatory treatment in schizophrenia. Prog. Neuropsychopharmacol. Biol. Psychiatry 42, 146-153 (2013).

65. Weiser, M., Burshtein, S. \& L, F. A randomized trial administering aspirin, minocycline or pramipexole vs placebo as add-on to antipsychotics in patients with schizophrenia or schizoaffective disorder. Neuropsychopharmacology 38, abstr S314-S446 (2012).

66. McAllister, C. G. et al. Increases in CSF levels of interleukin-2 in schizophrenia: effects of recurrence of psychosis and medication status. Am. J. Psychiatry 152 1291-1297 (1995).

67. Lin, A. et al. The inflammatory response system in treatment-resistant schizophrenia: increased serum interleukin-6. Schizophr. Res. 32, 9-15 (1998).
68. Zhang, X. Y., Zhou, D. F., Cao, L. Y., Wu, G. Y. \& Shen, Y. C. Cortisol and cytokines in chronic and treatment-resistant patients with schizophrenia: association with psychopathology and response to antipsychotics. Neuropsychopharmacology 30, 1532-1538 (2005).

69. Mondelli, $\mathrm{V}$. et al. Cortisol and inflammatory biomarkers predict poor treatment response in first episode psychosis. Schizophr. Bull. 41, 1162-1170 (2015).

70. Lodolce, J. P. et al. IL-15 receptor maintains lymphoid homeostasis by supporting lymphocyte homing and proliferation. Immunity 9, 669-676 (1998).

71. Mortier, E. et al. Macrophage- and dendritic-cell-derived interleukin-15 receptor alpha supports homeostasis of distinct CD8+ T cell subsets. Immunity 31, 811-822 (2009).

72. Gomez-Nicola, D., Valle-Argos, B., Pita-Thomas, D. W. \& Nieto-Sampedro, M Interleukin 15 expression in the CNS: blockade of its activity prevents glial activation after an inflammatory injury. Glia 56, 494-505 (2008).

73. Gomez-Nicola, D., Valle-Argos, B., Pallas-Bazarra, N. \& Nieto-Sampedro, M. Interleukin-15 regulates proliferation and self-renewal of adult neural stem cells. Mol. Biol. Cell. 22, 1960-1970 (2011).

74. Wu, X. et al. Interleukin-15 affects serotonin system and exerts antidepressive effects through IL15Ralpha receptor. Psychoneuroendocrinology 36, 266-278 (2011).

75. Pan, W. et al. Brain interleukin-15 in neuroinflammation and behavior. Neurosci. Biobehav. Rev. 37, 184-192 (2013).

76. Guyon, A. CXCL12 chemokine and GABA neurotransmitter systems crosstalk and their putative roles. Front. Cell. Neurosci. 5, 115 (2014).

77. Torrey, E. F. et al. Cytomegalovirus and schizophrenia. CNS Drugs 20, 879-885 (2006).

78. Regier, D. A. et al. Comorbidity of mental disorders with alcohol and other drug abuse. Results from the Epidemiologic Catchment Area (ECA) Study. JAMA 264, 2511-2518 (1990). 